\title{
Small interfering RNA-induced inhibition of epithelial cell transforming sequence 2 suppresses the proliferation, migration and invasion of osteosarcoma cells
}

\author{
JIE XIE, PENGFEI LEI and YIHE HU \\ Department of Orthopedics, Xiangya Hospital of Central South University, Changsha, Hunan 410008, P.R. China
}

Received June 2, 2014; Accepted February 11, 2015

DOI: $10.3892 /$ etm.2015.2306

\begin{abstract}
Osteosarcoma (OS) is the most common malignant tumor in bones. Although the five-year survival rate has improved to $\sim 60 \%$ for patients without metastasis, the prognosis remains poor for patients with metastatic OS. Epithelial cell transforming sequence 2 (ECT2) has been shown to act as an oncogene in human malignancies. More recently, ETC2 was shown to be involved in the development and progression of OS; however, the detailed role of ECT2 in the regulation of cellular biological processes in OS cells remains largely unknown. Therefore, it was investigated in the present study. It was found that the expression of ECT2 was notably increased in OS tissues when compared with that in matched normal adjacent tissues. Furthermore, it was established that the downregulation of ECT2 induced by transfection with ECT2-specific small interfering RNA effectively inhibited OS cell proliferation and induced cell apoptosis. Further investigation revealed that the inhibition of ECT2 expression suppressed OS cell migration and invasion, indicating that the overexpression of ECT2 promotes OS cell migration and invasion, while. In addition, western blotting results indicated that matrix metalloproteinases 2 and 9 may be involved in the ECT2-mediated OS cell invasion. In conclusion, the current study suggested that ECT2 acted as an oncogene in OS, and it may become a promising therapeutic target for the prevention and treatment of OS.

\section{Introduction}

Osteosarcoma (OS) is the most common malignant tumor of bone with a morbidity of $\sim 5$ cases per million. The prognosis with OS has improved following multi-agent chemotherapy, with a five-year survival rate at $\sim 60 \%$ for patients without

Correspondence to: Professor Yihe $\mathrm{Hu}$, Department of Orthopedics, Xiangya Hospital of Central South University, 87 Xiangya Road, Changsha, Hunan 410008, P.R. China

E-mail: csuhuyihe@163.com

Key words: osteosarcoma, epithelial cell transforming sequence 2, proliferation, apoptosis, invasion metastasis; however, the survival rate is poor for patients with metastatic OS (1). As dysfunctions of oncogenes or tumor suppressors are closely associated with the progression of OS, the development of effective molecular targets may show promise in the treatment of OS (2).

Epithelial cell transforming sequence 2 (ECT2), a guanine nucleotide exchange factor (GEF), is associated with Rho-specific exchange factors, and it has been shown to be involved in the regulation of cell cycle progression and cytokinesis $(3,4)$. It has also been suggested that ECT2 acts as an oncogene in human malignancies $(5,6)$. For instance, Murata et al (7) found that an abnormality in ECT2 occurred at a relatively early stage of lung adenocarcinogenesis, and suggested that ECT2 may be used as a novel biomarker for predicting the outcome of patients with lung adenocarcinoma. More recently, ECT2 was reported to be involved in OS $(8,9)$. Zhang et al (9) demonstrated that the messenger RNA (mRNA) expression level of ECT2 was increased in OS tissues compared with that in non-cancerous bone tissues, and was negatively correlated with the expression level of microRNA (miR)-223, which could bind to the 3'-untranslational region of ECT2 mRNA and thus suppress its protein expression. Additionally, it was found that the combined miR-223 downregulation and ECT2 upregulation was significantly associated with high tumor grade, poor response to chemotherapy, positive metastasis, recurrence of OS and poor prognosis, suggesting that the combined miR-223 downregulation and ECT2 upregulation may be used as a marker of poor prognosis in OS (9). Another study (8) investigated the role of miR-223 in the regulation of OS Saos-2 cell proliferation and cell cycle progression, and suggested that miR-223 was a tumor suppresser in OS and miR-223/ECT2 signaling had an inhibitory effect on OS cell cycle progression and proliferation; however, the detailed role of ECT2 in the regulation of OS cell biological processes, particularly for cell invasion, remains largely unknown.

The present study aimed to explore the role of ECT2 in the regulation of cell proliferation, apoptosis, migration and invasion in OS cells.

\section{Materials and methods}

Tissue specimen collection. The present study was approved by the Ethics Committee of Central South University (Changsha, China) and written informed consent was obtained. Eight 
primary OS samples and their normal matched adjacent tissues were collected at the Department of Orthopedics, Xiangya Hospital of Central South University. Tissues were immediately snap-frozen in liquid nitrogen following surgical removal.

Cell culture. Human OS cell lines, Saos-2, MG63 and U2OS, as well as human osteoblast cell line hFOB1.19 were obtained from the Cell Bank of Central South University. Cells were cultured in RPMI-1640 medium with $10 \%$ fetal bovine serum (FBS) at $37^{\circ} \mathrm{C}$ in a humidified incubator containing $5 \% \mathrm{CO}_{2}$.

Reverse transcription-quantitative polymerase chain reaction $(R T-q P C R)$ analysis. Total RNA was extracted from tissues or cells using TRIzol ${ }^{\circledR}$ (Life Technologies, Carlsbad, CA, USA), in accordance with the manufacturer's instructions. Expression of mRNA was examined using the standard SYBR Green RT-PCR kit (Takara, Otsu, Japan), in accordance with the manufacturer's instructions. The specific primer pairs were as follows: ECT2, sense: 5'-ACTACTGGGAGGACTAGCTTG-3'; and antisense: 5'-CACTCTTGTTTCAATCTGAGGCA-3'; glyceraldehyde-3-phosphate dehydrogenase (GAPDH) as an internal reference, sense: 5'-GGAGCGAGATCCCTCCAAAAT-3'; and antisense: 5'-GGCTGTTGTCATACTTCTCATGG-3'. The relative expression of mRNA was quantified using a $2^{-\Delta \Delta C}$ method. The amount of RNA analyzed per assay was $1 \mu \mathrm{g}$. The reaction was conducted in an ABI 7500 thermocycler (Life Technologies, Carlsbad, CA, USA).

Transfection. Cells were cultured to $70 \%$ confluence and resuspended in serum-free medium. Lipofectamine 2000 $0^{\mathrm{TM}}$ (Life Technologies) was used to perform transfection according to the manufacturer's instructions. Three groups of cells were established: Control group, cells cultured without any transfection; negative control (NC) group, cells transfected with non-specific small interfering RNA (siRNA); and ECT2 siRNA group, cells transfected with ECT2 siRNA. The siRNA was control siRNA-A (sc-37007, Santa Cruz Biotechnology, Dallas, TX, USA) and Ect2 siRNA (h) (sc-35259; Santa Cruz Biotechnology), respectively. Briefly, siRNA and Lipofectamine 2000 were diluted with serum-free medium. The diluted Lipofectamine 2000 was added to the diluted siRNA and incubated for $20 \mathrm{~min}$ at room temperature, and then added into the cell suspension. The cells were then incubated at $37^{\circ} \mathrm{C}$ and $5 \% \mathrm{CO}_{2}$ for $6 \mathrm{~h}$. Subsequent to that, the medium in each well was replaced by the normal serum-containing medium, and cultured for $24 \mathrm{~h}$ prior to the following assays.

Western blotting. Tissues or cells were solubilized in cold radio-immunoprecipitation assay lysis buffer. Proteins were separated with $12 \%$ SDS-PAGE, and transferred onto a polyvinylidene difluoride (PVDF) membrane, which was then incubated with Tris-buffered saline-Tween ${ }^{\circledast}$ containing 5\% milk at room temperature for $3 \mathrm{~h}$. The PVDF membrane was then incubated with the primary antibodies rabbit anti-ETC2 polyclonal antibody (1:100; ab123571; Abcam, Cambridge, MA, USA), rabbit anti-matrix metalloproteinase (MMP) 2 monoclonal antibody (1:50; ab51125; Abcam), rabbit antiMMP9 polyclonal antibody (1:50; ab38898; Abcam). and rabbit anti-GAPDH monoclonal antibody (1:50; ab181602; Abcam) at room temperature for $3 \mathrm{~h}$. Following washing three times with phosphate-buffered saline-Tween (PBST), the membrane was incubated with goat anti-rabbit IgG secondary antibodies (1:50; ab175781; Abcam) at room temperature for $40 \mathrm{~min}$. Chemiluminescent detection was performed using an electrochemiluminescence kit (Pierce Chemical, Rockford, IL, USA). The relative level of protein expression was analyzed using Image-Pro plus software (version 6.0; Media Cybernetics Inc., Rockville, MD, USA), and is represented as the density ratio versus GAPDH.

MTT assay. An MTT assay was used to evaluate the cell proliferation. Cells in each group were cultured in 96-well plates, where each well contained $100 \mu \mathrm{l}$ fresh serum-free medium with $0.5 \mathrm{~g} / 1 \mathrm{MTT}$. Following incubation at $37^{\circ} \mathrm{C}$ for $6,12,24$ and $48 \mathrm{~h}$, the medium was removed by aspiration and $50 \mu \mathrm{l}$ DMSO was added to each well. After incubation at $37^{\circ} \mathrm{C}$ for a further $10 \mathrm{~min}$, the absorbance of each sample at $570 \mathrm{~nm}$ was measured using a microplate reader (Bio-Rad, Hercules, CA, USA)

Apoptosis analysis, Flow cytometry (BD AccuriC6; BD Biosciences, Franklin Lakes, NJ, USA) was used to determine the cell apoptosis with an Annexin V-FITC Apoptosis Detection kit (Sigma-Aldrich, St. Louis, MO, USA), according to the manufacturer's instructions. Cells were harvested and washed with cold phosphate-buffered saline (PBS) twice. Subsequent to that, $1 \times 10^{6}$ cells were resuspended in $200 \mu \mathrm{l}$ binding buffer, $10 \mu \mathrm{l}$ Annexin-V-FITC and $5 \mu \mathrm{l}$ PI-PE were added, and the cells were incubated in the dark for $30 \mathrm{~min}$. Then, $300 \mu \mathrm{l}$ binding buffer was added followed by flow cytometric assay.

Transwell assay. Cell migration and invasion were analyzed by performing Transwell assays. A Corning ${ }^{\circledR}$ BioCoat $^{\mathrm{TM}}$ Matrige ${ }^{\circledR}$ Invasion Chamber with an $8.0-\mu \mathrm{m}$ PET membrane (Corning, Tewksbury, MA, USA) and Falcon ${ }^{\circledR}$ HTS 24 Well Multiwell Permeable Support system with an $8.0-\mu \mathrm{m}$ High Density PET Membrane (Corning) were used to investigate cell invasion and cell migration, respectively. In brief, cells were washed in cold-PBS, harvested and resuspended in serum-free Dulbecco's modified Eagle's medium (DMEM). For the invasion assay, $5 \times 10^{4}$ cells were added into the upper chamber, which was pre-coated with Matrigel. For the migration assay, $5 \times 10^{4}$ cells were added into the upper chamber, which was not pre-coated with Matrigel. Serum-free DMEM was then added to the upper chamber and DMEM medium containing $10 \%$ FBS as the chemoattractant was added to the lower chamber Following incubation for $24 \mathrm{~h}$ at $37^{\circ} \mathrm{C}$ with $5 \% \mathrm{CO}_{2}$, cells attached to the bottom of the membrane were fixed with $3.7 \%$ formaldehyde and then stained with crystal violet staining solution for $20 \mathrm{~min}$. A cotton swab was used to remove the cells that had not passed through the membrane. Five fields of the lower surface of the membrane were randomly selected under microscopy, and the cells on it were counted.

Statistical analysis. Data are presented as the mean \pm standard deviation. Student $t$-tests or one-way analysis of variance were used to analyze statistical data with Graphpad Prism 5 
A

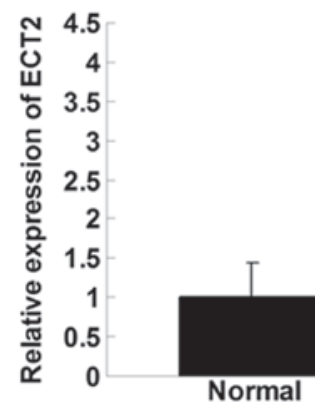

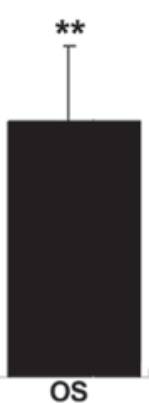

B

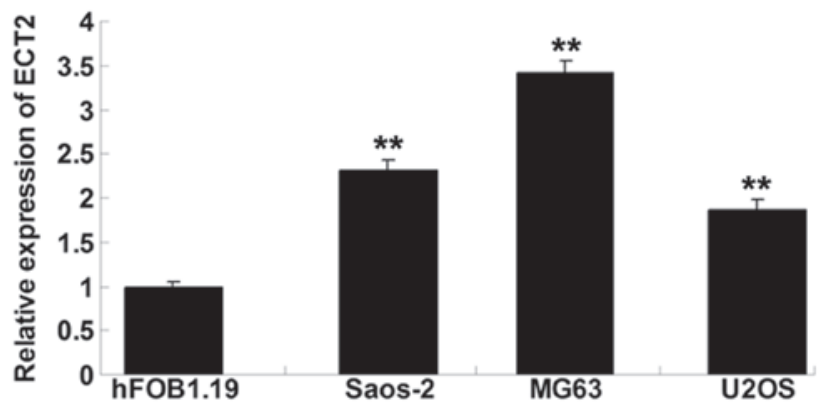

Figure 1. Expression levels of ECT2 were examined by carrying out reverse transcription-quantitative polymerase chain reactions in (A) OS tissues and their matched normal adjacent tissues $\left({ }^{* *} \mathrm{P}<0.01\right.$ vs. normal) and (B) in human OS cell lines, Saos-2, MG63, and U2OS, as well as human osteoblast cell line hFOB1.19 ( ${ }^{* *} \mathrm{P}<0.01$ vs. hFOB1.19). ECT2, epithelial cell transforming sequence 2; OS, osteosarcoma.

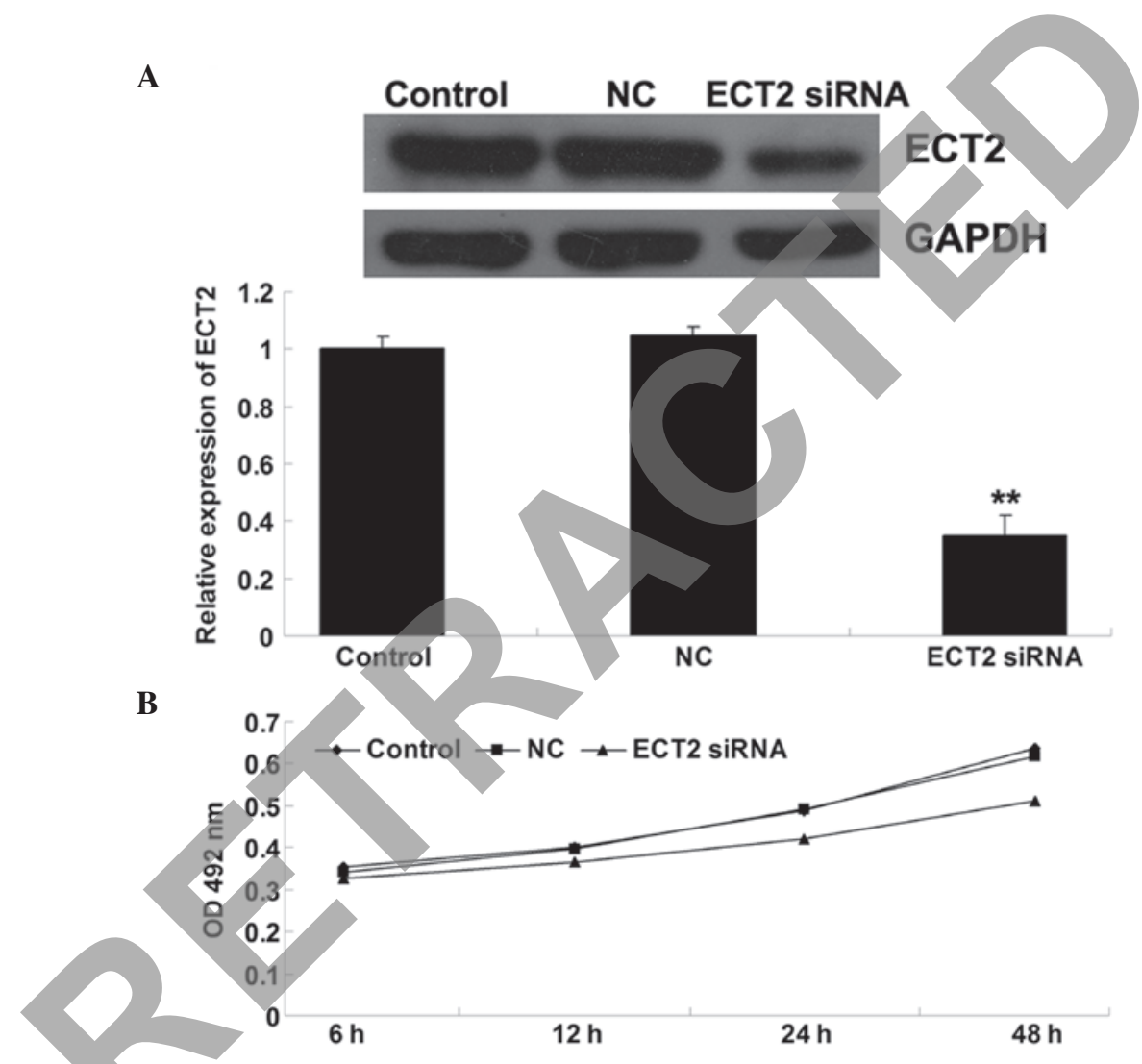

Figure 2. (A) Expression of ECT2 was examined by western blot analysis in MG63 cells with or without transfection with ECT2-specific siRNA (**P $<0.01$ vs. control). (B) A cell proliferation assay was performed in MG63 cells with or without transfection with ECT2-specific siRNA. Control, cells without any transfection; NC, cells transfected with non-specific siRNA; ECT2, epithelial cell transforming sequence 2; siRNA, small interfering RNA; OD, optical density; GAPDH, glyceraldehyde-3-phosphate dehydrogenase.

software (Graphpad, La Jolla, CA, USA). Compared with respective controls, $\mathrm{P}<0.05$ was considered to indicate statistical significance.

\section{Results}

Expression of ECT2 is notably increased in OS tissues and cell lines. To explore the role of ECT2 in OS, RT-qPCR was performed to determine the mRNA expression level of ECT2 in OS tissues as well as their matched normal adjacent tissues. As shown in Fig. 1A, the expression of ECT2 was notably upregulated in OS tissues, when compared with that in normal adjacent tissues. The expression of ECT2 in the three OS cell lines Saos-2, MG63 and U2OS was also examined. As shown in Fig. 1B, the expression level of ECT2 was increased in the OS cells compared with that in the normal osteoblast cell line hFOB1.19.

SiRNA-induced ECT2 downregulation inhibits OS cell proliferation. To further investigate the role of ECT 2 in the regulation of OS cell proliferation, MG63 OS cells were transfected with ECT2-specific siRNA. Following transfection, the effect of ECT-specific siRNA on the expression of ECT2 in MG63 cells was determined. As shown in Fig. 2A, ECT2-specific siRNA significantly inhibited ECT2 protein expression in MG63 cells, indicating that the transfection 

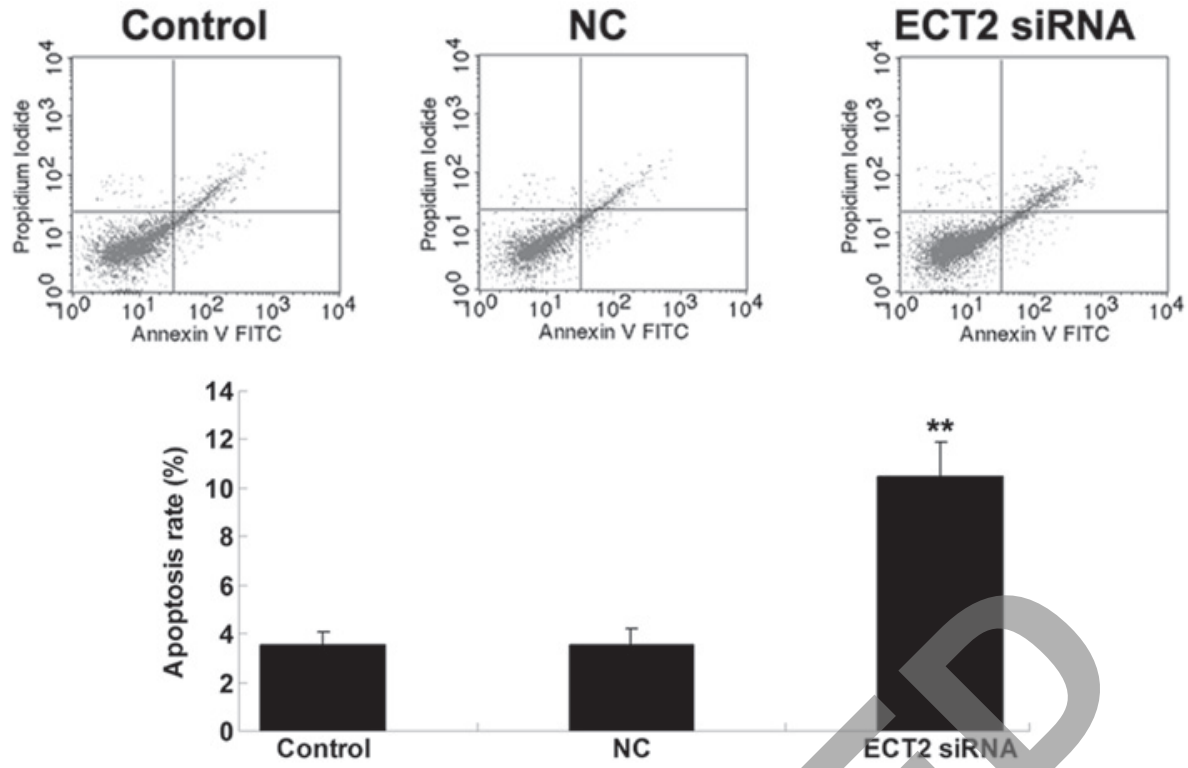

Figure 3. Cell apoptosis assay was performed in MG63 cells with or without transfection with ECT2-specific siRNA. Control, cells without any transfection; NC, cells transfected with non-specific siRNA; ECT2, epithelial cell transforming sequence 2; siRNA, small interfering RNA; FITC, fluorescein isothiocyanate. $\mathrm{P}<0.01$ vs. control.

A
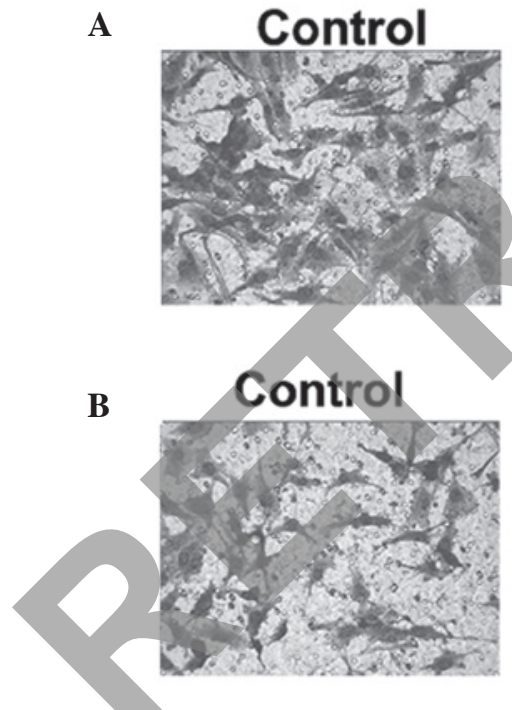

NC

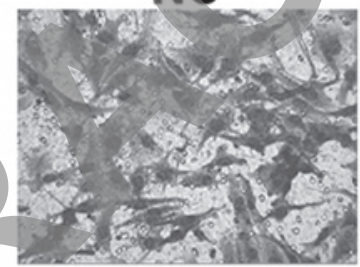

NC

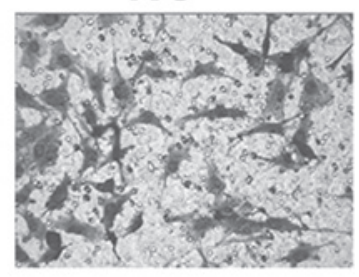

ECT2 siRNA

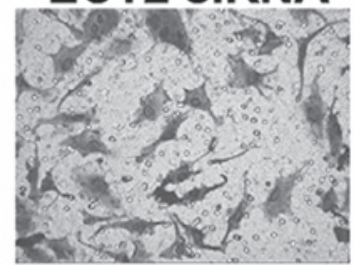

ECT2 siRNA

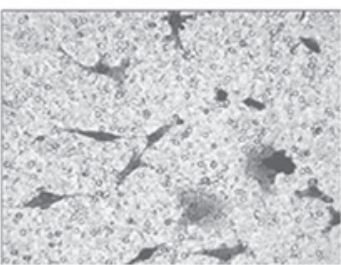

C
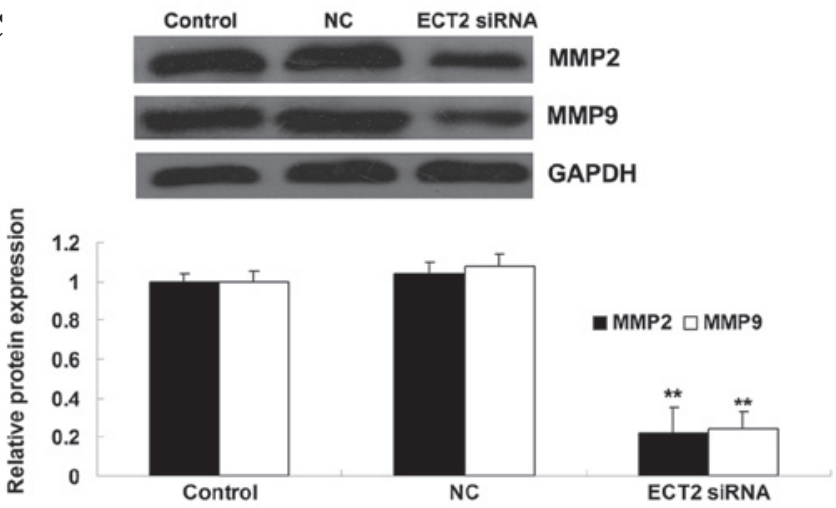

Figure 4. (A) Cell migration and (B) cell invasion assays were performed in MG63 cells with or without transfection with ECT2-specific siRNA. Magnification, x200. (C) Western blotting was performed to determine the protein expression of MMP2 and MMP9 in MG63 cells with or without transfection with ECT2-specific siRNA. GAPDH was used as an internal reference. Control, cells without any transfection; NC, cells transfected with non-specific siRNA; ECT2, epithelial cell transforming sequence 2; siRNA, small interfering RNA; GAPDH, glyceraldehyde-3-phosphate dehydrogenase; MMP, matrix metalloproteinase. ${ }^{* *} \mathrm{P}<0.01$ vs. control. 
efficiency was successful. An MTT assay was then performed to evaluate the proliferation of the cells. As shown in Fig. 2B, following transfection with ECT2-specific siRNA, the cell proliferation was notably reduced compared with that of the cells that did not undergo any treatment. This indicates that the siRNA-induced downregulation of ECT2 inhibited OS cell proliferation.

SiRNA-induced ECT2 downregulation promotes OS cell apoptosis. The effect of siRNA-induced ECT2 downregulation on MG63 cell apoptosis was then explored. As shown in Fig. 3, the cell apoptosis level was notably upregulated following transfection with ECT2-specific siRNA in MG63 OS cells, suggesting that siRNA-induced ECT2 downregulation promoted OS cell apoptosis.

ECT2 plays a promoting role in the regulation of OS cell migration and invasion. The role of ECT2 in the regulation of OS cell migration and invasion was also investigated. As shown in Figs. 4A and B, following the downregulation of ECT2 induced by siRNA, the cell migration and invasion were significantly decreased, when compared with those of control MG63 cells without any treatment. These data suggest that ECT2 plays a promoting role in the regulation of OS cell migration and invasion. Furthermore, the protein level of MMP2 and -9 in MG63 cells with or without transfection with ECT2-specific siRNA was examined. The data showed that siRNA-induced ECT2 downregulation led to a decrease in the protein levels of MMP2 and -9 in MG63 OS cells, suggesting that they were involved in the ECT2-mediated MG63 cell invasion (Fig. 4C).

\section{Discussion}

Although the five-year survival rate of patients with OS has improved, there remains a risk of relapse or metastasis even following curative resection. At the molecular level, most OS shows a marked alteration of gene expression profile; therefore, understanding the deregulation of oncogenes will be helpful in developing effective strategies for the treatment of OS (1). In the present study, the expression of ECT2 was shown to be significantly upregulated in OS tissues and cells compared with their matched normal adjacent tissues. The data further indicated that ECT2 played an oncogenic role in OS in vitro by promoting OS cell proliferation, migration and invasion, while inhibiting OS cell apoptosis.

ECT2 is a GEF for the Rho family of GTPases associated with cytokinesis (3). GEFs are able to activate the Rho GTPases in signal transduction, through catalyzing the exchange of guanosine diphosphate (GDP) for guanosine triphosphate (GTP). It has been reported that the copy number of ECT2-located 3q26 frequently increases in several cancers including head and neck, lung and cervical cancer, suggesting that a genomic imbalance may contribute to the upregulation of ECT2 in OS $(10,11)$. ECT2 has been considered to play an oncogenic role in several malignant tumors, including ovarian cancer, retinoblastoma, pancreatic ductal adenocarcinoma, cervical and colorectal cancers, oral squamous cell carcinoma, as well as OS $(9,12-17)$. In the present study, it was shown that the expression of ECT2 was significantly upregulated in OS tissues when compared with that in normal adjacent tissues. In addition, its expression was also upregulated in three OS cell lines. These data indicated that ECT2 played an important role in the development and progression of OS. The data were consistent with another study where the mRNA expression level of ECT2 was increased in OS tissues compared with noncancerous bone tissues (9); however, little is known about the mechanism of ECT2 in OS. To determine whether ECT2 function is relevant to OS progression, ECT2-specific siRNA was used to inhibit the expression level of ECT2 in OS cells. It was found that cell proliferation, migration and invasion were significantly reduced and cell apoptosis upregulated, suggesting that ECT2 was associated with OS progression.

It has been well-established that activated Rho GTPases are able to activate downstream effectors and influence numerous cellular biological processes, including cell survival, apoptosis, cell cycle progression and membrane trafficking, and they often cause tumorigenesis $(18,19)$. The role of ECT2 upregulation in other cancers has been widely demonstrated $(9,12-17)$; however, these studies mainly focused on its effect on cell cycle progression. For instance, Iyoda et al observed that ECT2 was notably upregulated in oral squamous cell carcinoma, and that the inhibition of ECT2 caused cell cycle arrest at the G1 phase, accompanied by the upregulation of the cyclin-dependent kinase (CDK) interacting protein/kinase inhibitory protein family of CDK inhibitors, as well as downregulation of cyclin D1, cyclin E and CDK4 (17). In the current study, it was shown that siRNA-induced ECT2 inhibition notably suppressed OS cell proliferation while promoting OS cell apoptosis. ECT2 has also been found to be involved in the regulation of cell cycle progression in OS cells. $\mathrm{Xu}$ et al (8) demonstrated that the downregulation of ECT2 in OS cells caused by miR-223 induced the arrest of cell cycle progression at the G1 phase, as well as the upregulated expression of p21 and p27, which are involved in the G1 blockade. Accordingly, it is suggested that the inhibition of cell proliferation and upregulation of cell apoptosis caused by siRNA-induced ECT2 downregulation may largely be attributed to cell cycle arrest.

Several studies have suggested that ECT2 participates in the regulation of cancer cell migration and invasion. For instance, ECT2 has been reported to be involved in the regulation of cell migration and invasion in glioblastoma cells, and depletion of ECT2 by siRNA has been demonstrated to suppress glioblastoma cell migration and invasion $(4,20)$. Sano et al (21) also found that ECT2 siRNA inhibited glioma cell invasion. In addition, another study reported that ECT2 played a role in the regulation of cell invasion in non-small cell lung cancer cells (22). To the best of our knowledge, the role of ECT2 in the regulation of cell migration and invasion, has never been reported in OS cells. In the present study it was shown that siRNA-induced ECT2 downregulation also notably inhibited OS cell migration and invasion, and the data suggested that MMP2 and -9 may act as downstream effectors in ECT2-mediated OS cell invasion. The role of ECT2 in OS cell invasion was, therefore, highlighted, and it may be associated with OS metastasis.

In conclusion, the expression of ECT2 was found to be significantly increased in OS tissues and cells. Additionally, the data demonstrated that ECT2 promotes cell proliferation, migration and invasion in OS cells. Accordingly, ECT2 may 
serve as a potential molecular target for the prevention and treatment of OS.

\section{References}

1. PosthumaDeBoer J, Witlox MA, Kaspers GJ and van Royen BJ: Molecular alterations as target for therapy in metastatic osteosarcoma: a review of literature. Clin Exp Metastasis 28: 493-503, 2011.

2. Liang W, Gao B, Fu P, Xu S, Qian Y and Fu Q: The miRNAs in the pathogenesis of osteosarcoma. Front Biosci (Landmark Ed) 18 788-794, 2013.

3. Tatsumoto T, Xie X, Blumenthal R, Okamoto I and Miki T: Human ECT2 is an exchange factor for rho GTpases, phosphorylated in G2/M phases and involved in cytokinesis. J Cell Biol 147: 921-928, 1999.

4. Salhia B, Tran NL, Chan A, et al: The guanine nucleotide exchange factors trio, Ect 2 and Vav3 mediate the invasive behavior of glioblastoma. Am J Pathol 173: 1828-1838, 2008.

5. Miki T, Smith CL, Long JE, Eva A and Fleming TP: Oncogene ect2 is related to regulators of small GTP-binding proteins Nature 362: 462-465, 1993

6. Fields AP and Justilien V: The guanine nucleotide exchange factor (GEF) Ect2 is an oncogene in human cancer. Adv Enzyme Regul 50: 190-200, 2010.

7. Murata Y, Minami Y, Iwakawa R, et al: ECT2 amplification and overexpression as a new prognostic biomarker for early-stage lung adenocarcinoma. Cancer Sci 105: 490-497, 2014.

8. Xu J, Yao Q, Hou Y, et al: MiR-223/Ect2/p21 signaling regulates osteosarcoma cell cycle progression and proliferation. Biomed Pharmacother 67: 381-386, 2013.

9. Zhang H, Yin Z, Ning K, Wang L, Guo R and Ji Z: Prognostic value of microRNA-223/epithelial cell transforming sequence 2 signaling in patients with osteosarcoma. Hum Pathol 45: 1430-1436, 2014.

10. Yang YL, Chu JY, Luo ML, et al: Amplification of PRKCI, located in $3 \mathrm{q} 26$, is associated with lymph node metastasis in esophageal squamous cell carcinoma. Genes Chromosomes Cancer 47: 127-136, 2008.
11. Hussenet T, Dali S, Exinger J, et al: SOX2 is an oncogene activated by recurrent $3 \mathrm{q} 26.3$ amplifications in human lung squamous cell carcinomas. PLoS One 5: e8960, 2010.

12. Wang Y, Hill KS and Fields AP: PKCiota maintains a tumor-initiating cell phenotype that is required for ovarian tumorigenesis. Mol Cancer Res 11: 1624-1635, 2013.

13. Nalini V, Segu R, Deepa PR, Khetan V, Vasudevan M and Krishnakumar S: Molecular insights on post-chemotherapy retinoblastoma by microarray gene expression analysis. Bioinform Biol Insights 7: 289-306, 2013.

14. Samuel N, Sayad A, Wilson G, et al: Integrated genomic, transcriptomic and RNA-interference analysis of genes in somatic copy number gains in pancreatic ductal adenocarcinoma. Pancreas 42: 1016-1026, 2013.

15. Vazquez-Mena O, Medina-Martinez I, Juarez-Torres E, et al: Amplified genes may be overexpressed, unchanged, or downregulated in cervical cancer cell lines. PLoS One 7: e32667, 2012.

16. Jung Y, Lee S, Choi HS, et al: Clinical validation of colorectal cancer biomarkers identified from bioinformatics analysis of public expression data. Clin Cancer Res 17: 700-709, 2011.

17. Iyoda M, Kasamatsu A, Ishigami T, et al: Epithelial cell transforming sequence 2 in human oral cancer. PLoS One 5: e14082, 2010.

18. Etienne-Manneville S and Hahl A: Rho GTPases in cell biology. Nature 420: 629-635, 2002.

19. Boettner B and Van Aelst L: The role of Rho GTPases in disease development. Gene 286: 155-174, 2002.

20. Fortin SP, Ennis MJ, Schumacher CA, et al: Cdc42 and the guanine nucleotide exchange factors Ect 2 and trio mediate Fn14-induced migration and invasion of glioblastoma cells. Mol Cancer Res 10: 958-968, 2012.

21. Sano M, Genkai N, Yajima N, et al: Expression level of ECT2 proto-oncogene correlates with prognosis in glioma patients. Oncol Rep 16: 1093-1098, 2006.

22. Justilien V, Jameison L, Der CJ, Rossman KL and Fields AP: Oncogenic activity of Ect 2 is regulated through protein kinase C iota-mediated phosphorylation. J Biol Chem 286: 8149-8157, 2011. 